way yielded $1 / 10$ as much, $0.00063 \%$. In this case also the proportion of gas found in pure metal is only $1 / 7$ that found by Guye and Germann.

The foregoing figures are based on the assumption that the iodine and the silver are responsible for all the gas evolved and that the gas is as heavy as air.

The likelihood has been pointed out that a portion of the gas obtained either was liberated from the walls of the apparatus or diffused through the reaction tube. Furthermore in the case of silver at any rate a large portion of the gas was found to be hydrogen. Therefore there is good reason to believe that the real percentages are smaller than those given above.

3. Even when maximum corrections are applied, the atomic weights of silver and of elements referred to silver are affected in the most unfavorable cases by only 0.002 unit.

A grant from the Wolcott Gibbs Fund of the National Academy of Sciences has materially aided the prosecution of this investigation.

Cambridge 38, MassachusetTs

[CONTRIBUtion fRom the T. JeFFERSON COOLIDGE, JR, Chemical Laboratory of HARVARD UNIVERSITY]

\title{
THE PURITY OF ATOMIC WEIGHT SILVER. II. SOLID IMPURI-
} TIES

\section{By Gregory Paul Baxter}

Received December 28, 1921

The preparation of silver of the highest purity for purposes of atomic weight comparison has been the subject of many investigations, the most recent of which is that of Richards and Wells, ${ }^{1}$ who found that the processes of precipitation as silver chloride, crystallization of silver nitrate, precipitation of the metal from solution by ammonium formate, ${ }^{2}$ and electrolytic transport of the metal from a dissolving anode through a concentrated solution of silver nitrate, ${ }^{3}$ are all effective in removing metallic impurities. In investigations in the Chemical Laboratories of Harvard University various combinations of these methods have been used both before and since the investigation of Richards and Wells, and the products have been found to yield identical results within the experimental error. ${ }^{4}$

The final treatment of the silver before weighing has consisted almost

I Richards and Wells, Carnegie Inst. Pub., 28, 16 (190z); This Journal, 27, 459 (1905).

2 Stas, "Oeuvres Complètés," vol. 3, p. 40.

3 Abrahall, J. Chem. Soc., 61, 660 (1892).

4 Guye has recently questioned the freedom of such material from metallic impurities. J. chim. phys., 15, 554 (1917). 
invariably of fusion on a boat of carefully purified lime, usually in a current of hydrogen, ${ }^{5}$ but sometimes in a vacuum. If the silver has been deposited from a silver nitrate solution, fusion in a vacuum was found by Richards and Wells to be insufficient, for the residual metal seems to retain a small amount of oxygen coming from the included pockets of silver nitrate solution. ${ }^{6}$ If electrolytic crystals are fused in hydrogen, however, the latter difficulty disappears, and this process, namely, fusion of the metal in a current of hydrogen on a boat of pure lime, has been utilized exclusively in recent years. When thus fused, the buttons, although bright, are coated with specks of adherent lime which must be removed by etching with dil. nitric acid several times. In this way a layer of silver of considerable thickness is removed. The final product, after being washed and dried is then heated to about $500^{\circ}$ in a vacuum.

Naturally the possibility exists of the presence of calcium and accluded hydrogen in the silver. The first point was considered very carefully by Richards and Wells who were unable to detect calcium by a wet process in a 10g. sample of silver which had been fused upon lime. ${ }^{7}$ Their experiment showed that the metal certainly contained less than $0.0001 \%$ of calcium.

Furthermore, Stas concluded that silver which had been fused and cooled in hydrogen does not contain more than $0.0004 \%$ of this element, and the experiments recorded in the preceding paper prove that the total gaseous impurity in the silver in question is less than $0.0006 \% .^{8}$ This quantity is barely significant in work of the utmost accuracy at the present time.

Since the spectroscope is a most uncompromising and sensitive instrument for the detection of metallic impurities and because of the success which has attended spectroscopic examination of pure metals for exact purposes in this Laboratory in recent years, ${ }^{9}$ a careful spectroscopic examination of several specimens of very pure silver has been carried out. To do this both the spark and the arc spectra between electrodes of the purest silver have been photographed and compared with spectra of various possible impurities produced under exactly similar conditions. Several different samples of silver which have been used in recent atomic weight comparisons have been examined.

One group of specimens had been subjected to essentially the same processes.

s Baxter, Proc. Am. Acad., 39, 249 (1903).

${ }^{\text {B }}$ Ref. 1, p. 23.

${ }^{7}$ Ref. 1, p. 22.

'See also: Graham, Jahresb., 1866, 51. Neumannn and Streintz, Monatsh., 12, 642 (1891). Baxter, Am. Chem. J., 22, 362 (1899).

\& Baxter and Hartmann, ThIs Journal, 37, 116 (1915). Baxter and Grover, ibid., 37, 1032, 1050 (1915). Baxter and Grose, ibid., 38, 859, 869 (1916). 
The first step consisted of double precipitation of silver chloride, with either intermediate reduction by means of sugar and sodium hydroxide, and solution of the metal in nitric acid, or solution of the first precipitate of silver chloride in ammonia and reprecipitation with nitric acid. After the chloride had been reduced with sugar and the metal fused on charcoal, the buttons were clearised and dissolved in nitric acid, and the solution was heated with an excess of ammonium formate. The washed precipitated silver was again fused in air on a porcelain crucible lined with the purest lime, and then the buttons were made the anode under a concentrated solution of silver nitrate made from one of the buttons. The electrolytic crystals were fused in a current of the purest hydrogen on a boat of pure lime, contained in a porcelain tube. Finally the buttons were several times etched with small portions of dil. nitric acid, and after thorotugh washing and drying in the air were heated in a hard glass or quartz tube to about $500^{\circ}$. Four different specimens of this variety of silver were examined. One had already been used by Baxter, Moore and Boylston, ${ }^{10}$ and three others had been prepared for work at present under way by Messrs. O. J. Stewart and Muneo Tanj and Dr. P. F. Weatherill.

In the other group of specimens, crystallization of silver nitrate from dil. nitric acid was followed by precipitation of the metal with ammonium formate, and the product was fused in hydrogen on lime as described above. One of these specimens had been used by Richards and Wells ${ }^{1}$ and had been crystallized 15 times as silver nitrate before precipitation with ammonium formate. The other had been used by Richards and Willard ${ }^{11}$ and had been crystallized 5 times as silver nitrate before the formate precipitation. It is to be noted that one of the chief differences between the two groups is that in the former case, the material before the fina: fusion consisted of fairly large electrolytic crystals, in the latter case of finely divided precipitated metal.

The spectrograms were taken with a Féry quartz spectrograph, which by means of a diaphragm permits the close juxtaposition of successive spectra without involving any movement and therefore lateral displacement of the film holder. The spark was produced by means of a half kilowatt wireless transformer, actuated by the 110 volt a. c. lighting current, with a condenser parallel with the spark and with sufficient self inductance in series with the spark to eliminate the air lines almost completely. In character the spectrum fell midway between an arc spectrum and that produced with the usual high tension interrupting coil. The arc was produced by stepping down with resistance a 110 volt d. c. current so that approximately 15 amperes passed between the points. The electrodes, which weighed 5 or more grams each, were held between the points of clean steel pincers in such a way that the arc or spark did not approach the steel.

The silver was compared with the following metals: magnesium, zinc, cadmium, mercury, aluminum, chromium, iron, nicke1, copper, gold, platinum, tin, lead, calcium and bismuth. This list includes all the metals found in commercial silver by de Gramont. ${ }^{2}$

Although the exposures were prolonged, two minutes or more in every

io Baxter, Moore and Boylston, This Jovrwal, 34, 260 (1912).

1 Richards and Willard, Carnegie Inst, Pub., 125, 16 (1910); 'This Journaz, 32, $18(1910)$.

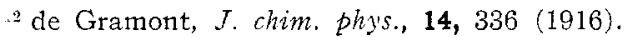


case, not the smallest trace of any one of these metals could be discovered, with the sole exception of calcium. Calcium lines were faintly visible in every one of the specimens with slightly varying intensity. Comparison of the spectrograms with others of metals containing known proportions of impurity indicates that the proportion of calcium in even the least pure specimen is of the order of one part in a million. This is in entire accord with the experiment of Richards and Wells mentioned above. liurthermore, in the specimen prepared by Richards and Willard the two strongest lines of silicon are very faintly visible. Here again the proportion seems to be extremely small, not greater than, if as great as, the proportion of calcium.

Nevertheless, a further attempt was made to discover the proportion of calcium as follows.

All the buttons which had been used in the spectroscopic experiments, $78 \mathrm{~g}$. in all, were once more slightly etched with dil. nitric acid in order to remove surface impurities acquired during handling. This could hardly have altered the composition of the silver since all the material had previously been etched several times in the same way. The material was then dissolved in a platinum dish in nitric acid which had been distilled through a quartz condenser, and about half of the silver was next deposited electrolytically chiefly upon the dish which was made the negative pole, but partly as peroxide on the anode. The electrolyte was evaporated nearly to dryness in another platinum dish over an electric stove, and after solution of the residue in the purest water about half the remaining silver was deposited electrolytically.

Evaporation and a third electrolysis removed all but traces of the silver. The final electrolyte was evaporated to dryness and the solution of the residue in water was saturated with hydrogen sulfide to remove the last traces of silver. After filtration, a slight excess of ammonia, in the form of gas, was introduced to remove a trace of iron which originated in the steel pincers used for holding the buttons during the spectroscopic experiments. The volume of the solution at this stage was about $20 \mathrm{cc}$. Filtration and evaporation to dryness in a weighed platinum crucible followed, and the residue was heated to about $600^{\circ}$ to expel ammonium salts. The residue weighed $0.12 \mathrm{mg}$. When dissolved in a few drops of very dilute nitric acid and made alkaline with ammonia the solution gave a barely visible precipitate with ammonium oxalate in a volume of about $1 \mathrm{cc}$, while $0.03 \mathrm{mg}$. of calcium under similar conditions gave a distinctly heavier precipitate.

The proportion of calcium in the silver therefore did not exceed $0.00004 \%$ on an average, somewhat less than the estimate made above, and in entire accord with the experiments of Richards and Wells.

\section{Summary}

It is evident therefore that in the present state of the most accurate chemical analysis the purity of these specimens of silver is considerably more than sufficient. 